\title{
Associating Factors with Outcome (Modified Ranking Scale) of Spontaneous Intracerebral Haemorrhage Cases Attending At Tertiary Care Hospital, Udaipur (Rajasthan)
}

\author{
Dr Rishi Ram Meena ${ }^{1}$, Dr O. P Meena, ${ }^{2}$ Dr Rashmi Gupta ${ }^{3}$ \\ ${ }^{I}$ Resident of Department of General Medicine \\ ${ }^{2}$ Professor and Unit Head of Department of General Medicine, RNT medical college, Udaipur \\ ${ }^{3}$ Assistant Professor, Department of community medicine, JLN Medical College, Ajmer \\ Correspondence Author: \\ Dr Rashmi Gupta \\ Assistant Professor, Department of community medicine \\ JLN Medical College, Ajmer \\ Email:drrashu53@rediffamil.com
}

\begin{abstract}
Study Question : spontaneous intracerebral hemorrhage (SICH) remains the deadliest and most disabling form of stroke.

Objective: to find out clinical profile, identifying definite risk factors, to assess the utility of (ICH) Score and Intra Cerebral Haemorrhage-Graded Scale (ICH-GS) as tools to predict outcome and to apply Siriraj score and the Guy Allen score to classify patients as infarcts and haemorrhages based on the clinical presentation as predicted by the scores.
\end{abstract}

Methodolgy: Single center, prospective, descriptive study, Department of Internal Medicine/Neurology RNT Medical College, Udaipur from December 2012-13 conducted in 50 patients of Spontaneous Intracerebral Haemorrhage as confirmed by CT of the brain were selected .Patients on thrombolytics, anti-coagulants, Antiplatelets, Traumatic haemorrhage, Venous infarct with hemorrhagic Transformation, Aneurysmal bleeding, Bleeding disorders, Coagulation disorder, Malignancy, Toxic cause were excluded. Approval from ethical committee were obtained. The initial assessment on admission using Glasgow Coma Scale (GCS), the National Institute of Health Stroke Scale (NIHSS), (ICH) Score and the ICH Grading Scale (ICH-GS) were calculated. The Siriraj score and Guy Allen score, (CT) Scanning of the Brain, the volume of the bleed was calculated using the $A B C / 2$ technique. The bedside $A B C / 2$ method, the $C T$ slice with the largest area of haemorrhage was identified. Outcome was evaluated using the Modified Ranking Scale (MRS)The data thus obtained were analysed with the help Microsoft Excel 2007 and statistical software PRIMER.

Result : Out of 50 patients, mean age of 63.46 years, Male :Female ratio was 1.27:1. No significant association of MRS was observed with gender and age hypertension SBP and DBP pulse pressure the site of bleed. All 50 patients had a supra-tentorial site of bleed. Cases with IVH, Glasgow Coma Scale on admission volume of the bleed of intracerebral haemorrhage ICH and ICH-GS were significantly associated with Outcome P<0.05S) The most common cause of death was respiratory depression, followed by aspiration pneumonitis which occurred in $41 \%$ of patients who developed complications.

Keywords: spontaneous intracerebral hemorrhage, Modified Ranking Scale

\section{Introduction}

Of all the cerebrovascular diseases, brain haemorrhage is the most dramatic and from ones has been surrounded by 'an aura of mystery and inevitability'.Intracerebral hemorrhage (ICH) accounts for 20- 30\% of all cases of stroke among Asian population approximately twice higher than that in the West ${ }^{1}$ An early and accurate prediction of ICH outcome in the emergency department is crucial for decision-making, as well as in assessing patient prognosis. ${ }^{[2,3]}$ With the arrival of CT of the brain, diagnosis of ICH has been taken from the age of calculated speculation with details of clinical features, angiograms and 'bloody taps' to the present day of arrival of a definitive diagnosis in a matter of minutes.

A significant reason for the decreasing trend of mortality in the ICH patients in the industrialized countries have also been due to the identification of the factors which might adversely affect the outcome, stratifying patients and instituting prompt acute stroke care. Though medical management of ICH has been the way, surgical management has been known .Though no definite guidelines exist to decide between the two lines 
of management, the increasing availability of minimally invasive techniques like stereo- tactic aspiration may make this modality indispensable in the days to come.

But all is not well for a country like India, where there still exist pockets where CT brain is unheard of, stroke care centers unimaginable, and supply almost always short of demand. Thus, this study was designed to find out clinical profile, identifying definite risk factors, making prompt and accurate diagnosis, stratifying patients according to outcome predictors, to assess the utility of (ICH) Score and Intra Cerebral HaemorrhageGraded Scale (ICH-GS) as tools to predict outcome and thereby ensuring prompt referral of deserving critical patients to tertiary centers for intensive management, may be the need of the hour as wait to embrace the newer advances into our management protocol.

\section{Materials And Methods}

Single center, prospective, descriptive study, In-patients department, Department of Internal Medicine/Neurology, MBGH RNT Medical College, Udaipur from December 2012-13 conducted in 50 patients of Spontaneous Intracerebral Haemorrhage as confirmed by computed tomography of the brain were selected after taking informed consent from the patients and from the closest relative in case of patients in altered sensorium. Patients on thrombolytics, anti-coagulants, Antiplatelets, Traumatic haemorrhage, Venous infarct with hemorrhagic Transformation, Aneurysmal bleeding, Bleeding disorders, Coagulation disorder, Malignancy, Toxic cause i.e. snake bite were excluded. Approval from ethical committee were obtained.

Patients' demographic, social, economic and medical details symptoms preceding the present illness were recorded in the proforma .

The initial assessment on admission included assessment of level of consciousness using the Glasgow Coma Scale (GCS), blood pressure measurement, neurological deficit assessment using the National Institute of Health Stroke Scale (NIHSS), (ICH) Score and the ICH Grading Scale (ICH-GS) were calculated. The Siriraj score was calculated using details of the clinical presentation on admission and the Guy Allen score was calculated 24 hours after admission to differentiate patients into the subtypes of strokes. Computed Tomography (CT) Scanning of the Brain, the volume of the bleed was calculated using the ABC/2 technique. The bedside $\mathrm{ABC} / 2$ method, the CT slice with the largest area of haemorrhage was identified.

Outcome was evaluated on the day of discharge (or earlier in case of death) and functional disability graded using the Modified Ranking Scale (MRS). This score assesses functional independence and impact on activities in daily living and grades patients from 0 (no symptoms) to 6 (death). In the study a score more than 4 is taken as poor outcome and a score lesser than or equal to 4 as good outcome. The data thus obtained were analysed with the help Microsoft Excel 2007 and statistical software PRIMER.

\section{Results}

Out of 50 patients, 28(56\%) were male and 22(44\%) were female with M:F ratio 1.27:1. On comparing gender and outcome in the form of Modified Ranking Score MRS ,Fifteen (53.57\%) out of 28 male are with good outcome and $13(46.43 \%)$ male with bad outcome and 8(36.36\%) out of 22 female are with good outcome and $14(63.64 \%)$ female with bad outcome. ( $\mathrm{P}=0.226 \mathrm{NS})$. The age of patients in the study ranged from 35 to 91 years with a mean of 63.46 years. The mean age of the female patients was $62.82 \mathrm{yrs}$ and that of male patient 63.96 yrs. Among 50 cases, 39 cases were below 80 yr and 11 cases above 80 yrs. Below 80 yrs, 18(46.15\%) out of 39 had good outcome and 21(23.85\%) had bad outcome. Above 80 yrs 5(45.45\%), out of 11 had good outcome and $6(54.54 \%)$ had bad outcome. $(\mathrm{P}=0.96 \mathrm{NS})$ History of Diabetes Mellitus was obtained in 1 patients accounting for $2 \%$ of the group. no statistically significant association was obtained between the history of Diabetes Mellitus and the outcome.

$8(44.44 \%)$ out of 18 hypertensive cases, had good outcome and $10(55.55 \%)$ had bad outcome. 15 (46.88\%) out of 32 non hypertensive cases had good outcome and 17 (53.12\%) had bad outcome. Thirty six\% patients gave a history of hypertension and this was not associated with a statistically significant association with outcome.

$6(46.15 \%)$ out of $13(26 \%)$ smoker cases had good outcome and 7(53.84\%) bad outcome.16(43.24\%) out of 37 non smoker cases had good outcome and $21(56.76 \%$ ) bad outcome with $\mathrm{P}=0.85 \mathrm{NS}$ History of alcohol intake was observed $6 \%$, good outcome was observed in $75 \%$ and $1(25 \%)$ had bad outcome. 20(43.47\%) out of 46 cases are non alcoholic, had good outcome and 26(56.52\%) had bad outcome .On comparing this information with the outcome, no statistically significant association as found. Other co-existent illnesses were identified in $7(14 \%)$. There was no statistically significant association with the presence of these illnesses with the outcome. The systolic blood pressures measured on admission was found to be in the range of $30-260 \mathrm{~mm}$ of $\mathrm{Hg}$ with a mean value of $170 \mathrm{~mm}$ of $\mathrm{Hg}$. SBP > $160 \mathrm{~mm}$ of $\mathrm{Hg}$ was in 44 (88 \%) of patients.20/44 (45.45\%) systolic hypertensive cases had good outcome and $24 / 44(54.54 \%)$ had bad Outcome. $4 / 6(75 \%)$ out of 6 nonsystolic hypertensive cases had good outcome and $2(25 \%)$ had bad outcome.(P>0.05NS) 
The diastolic blood pressure was 104( Range $75-150 \mathrm{~mm}$ of $\mathrm{Hg}$ ). The diastolic pressure (> $100 \mathrm{~mm}$ of $\mathrm{Hg}$ ) was in $23(46 \%)$ of patients. 14/27 (51.85\%) diastolic hypertensive cases had good outcome and $13(48.15 \%)$ had bad outcome and $9 / 23$ (39.13\%) non-diastolic hypertensive cases had good outcome and $14(60.87 \%)$ had bad outcome(P>0.05NS) $.34(68 \%)$ had wide pulse pressure.15/34 (44.11\%) cases had good outcome with wide pulse pressure and $19(55.89 \%)$ had bad outcome and 8/16 (50\%) out of 16 cases had good outcome with wide pulse pressure and $8(50 \%)$ had bad outcome.(P $>0.05 N S)$. All 50 patients had a supratentorial site of bleed. No statistically significant correlation was found between the site of bleed and outcome. intraventricular extension of the bleed was observed in $24(48 \%)$. 17/24(70.83\%) cases with IVH had significantly more worse outcome as compared to $10 / 26(38.46 \%)$ cases without IVH on the Modified Ranking Score. $(\mathrm{P}=0.02 \mathrm{~S})$

Glasgow Coma Scale on admission and was graded as per severity. All 19 cases with GCS 3-8 had bad outcome. 5/13(38.46\%) with GCS 8-12 had good outcome and 8(61.54\%) had bad outcome while all 18 cases with GCS 12-15 had good outcome. $(\mathrm{P}<0.05 \mathrm{~S})$ The volume of the bleed of intracerebral haemorrhage $26.91 \mathrm{ml}$.( Range 0.495 to 198.9)as calculated by the $\mathrm{ABC} / 2$ method from the CT scan .Among 50 study cases,18(36\%) cases had ICH volume $>30 \mathrm{ml}$ and $32(64 \%)$ had $<30 \mathrm{ml} .1(5.56 \%)$ out of 18 cases of ICH volume $>30 \mathrm{ml}$ had good outcome and $17(94.44 \%$ ) had bad outcome.22(68.75\%) out of 32 cases of ICH volume $<30 \mathrm{ml}$ had good outcome and $10(31.25 \%)$ had bad outcome. $(\mathrm{P}<0.001 \mathrm{~S})$

The Intra Cerebral Haemorrhage (ICH) score was compared with the outcome as measured by the modified Ranking Score on the day of discharge to reveal a statistically significant association between increasing score and worsening outcome. Among the 50 case study, 8(16\%) cases with ICH score zero and $42(84 \%)$ with $\mathrm{ICH}$ score $\geq 1$ as compared to all 8 cases with ICH score zero had good outcome while $15 / 42$ (35.71\%) cases had good outcome cases with ICH score $\geq 1$. $(\mathrm{P}<0.001 \mathrm{~S})$

The ICH-GS scoring was calculated on the admission day and was compared with the outcome on the day of discharge to reveal a positive statistically significant correlation with the increasing score and the worsening outcome. $27(34 \%)$ with ICH-GS $\leq 6$ and remaining $33(66 \%)$ with ICH-GS $>6$ while $15 / 17(88.2 \%)$ with ICH-GS $\leq 6$ had good outcome and $2(11.8 \%)$ had bad outcome. As compared to $6 / 33(19.2 \%)$ with ICHGS $>6$ had good outcome and 27/33(80.8\%) had bad outcome. $(\mathrm{P}<0.001 \mathrm{~S})$ The patients were evaluated on their last day in the hospital for the outcome using the Modified Ranking Score based on the functional ability and graded from 0 (no disability) to 6 (death) with increasing disability on the scale. The most common cause of death was respiratory depression, followed by aspiration pneumonitis which occurred in $41 \%$ of patients who developed complications.

The Siriraj score was calculated at the time of admission. It was found that the score predicted haemorrhage with an accuracy of 50\% in this study. Using the clinical features and symptoms, the Guy Allen score was calculated 24 hours after admission and used to stratify patients into haemorrhage $(>+14)$, infarct $(<+4)$ or equivocal.

\section{Discussion}

The mean age of patients in the study group was found to be $63.46 \mathrm{yrs}$, slightly higher in the females (62.82yrs) than the males (63.96 yrs). The increasing incidence of stroke with increasing age has been demonstrated convincingly by the Framingham study ${ }^{4}$. In this study, an increasing trend was noticed in the age group of 45 to 64 years among both sexes. A steady slope was noticed in the incidence in ICH in the age group over $65 \mathrm{yrs}$ and this might be attributable to the smaller subset of this population as the average life expectancy of the Indian population is around 64 years, a finding akin to that of the Framingham study ${ }^{4}$. The ratio of male to female patients was 1.3:1. This is in contrast to the findings of the Framingham study where equal distribution was found between the two sexes. This might be partly explained by the fact that medical attention is sought more often in the male patients than the female patients in our country.

$36 \%$ of the patients had a history of hypertension with more than two thirds of the hypertensive patients on irregular treatment. Another $10 \%$ of the non hypertensive patients had electrocardiographic evidence of left ventricular hypertrophy $(\mathrm{LVH})$, which is a surrogate marker of chronic hypertension. Though Hypertension is the single most important risk factor for intracerebral haemorrhage ${ }^{5,6}$, evidence for its presence was found only in around $50 \%$ of patients..

Though Diabetes Mellitus hasn't been known directly as a risk factor for ICH, it has been noted to be a known to increase the risk of a stroke in patients who might be hypertensives ${ }^{7}$, Arboix et al identified history of Diabetes Mellitus as an independent predictor of worse outcome ${ }^{8}$.

History of ingestion of $>60 \mathrm{~g}$ of alcohol was obtained in $6 \%$ and history of smoking on $26 \%$ of patients. Though history of alcohol intake is known to be a predisposing factor in intracerebral haemorrhage, its presence in less than $40 \%$ of patients might be due to the socio-cultural factors which might force patients or patients' care takers from withholding this information to medical personnel. 
Systolic blood pressure was found to be elevated to $>160 \mathrm{~mm}$ of $\mathrm{Hg}$ is in $>88 \%$ on the day of admission and persistently elevated in $70 \%$ of the patients in the following 24 hours. This might not be representative of all the hypertensive patients in the study group as the phenomenon of acute reactional hypertension is a recognized event in the acute settings of $\mathrm{ICH}^{3}$.Nevertheless, the need for monitoring hypertension till the day of discharge and appropriate treatment is imperative. The mortality rate for intracerebral haemorrhage is between $32-50 \%^{5,6}$, though in this study group the mortality rate was noticed to be $34 \%$. This could possibly be explained by the fact that a significant proportion of severely disabled patients went home against medical advice thereby the eventual course could not be followed up.

The most common cause of death was noted to be respiratory depression which was usually manifest by the first 48 hours followed by aspiration pneumonitis. Extension of haematoma was identified in 3 patients with worsening of sensorium as the clinical manifestation. This complication might have been underestimated in this study as repeat CT scans were taken only in those patients in whom this complication was strongly considered and not the others due to financial constraints.

Siriraj score and the Allen score, could correctly Identify haemorrhages based on clinical scenario in $58 \%$ and 50\% respectively. Combination of the above two scores did not have a better result. The chances of incorrectly identifying haemorrhagic patients as having infarcts were $20 \%$ for Siriraj score and $28 \%$ in the Allen score. These findings are akin to those of the studies conducted in India by Kochar et al ${ }^{9}$ though there are numerous studies with findings of accuracyof over $70 \%{ }^{10.11}$.

All the variables discussed above were tallied with the outcome as assessed by the modified Ranking Score to reveal the following details. Age has been identified to be a predictor of worse outcome in a few studies 12,13 and less consistently in the other studies. In this study, age was identified to be a highly significant independent predictor of worse outcome. The cut off value of age $>80$ yrs has been used by Claude Hemphill et al ${ }^{14}$, a lower cut off for age would seem more appropriate for an Indian population with an average life span of 64 years. Thereby, age > 65 yrs was used and was found to have highly significant correlation in predicting worse outcome.

GCS score has consistently been found to have a positive correlation in worse outcome in patients with stroke despite the shortcomings faced in a dysphasic patients. ${ }^{15.16}$ In this both GCS and NIHSS scores can independently predict outcome after a haemorrhagic stroke.

Volume of intra-cerebral haemorrhage, has been consistently shown to have a positive correlation especially in the supra-tentorial bleeds similar with this study ${ }^{17,}{ }^{18}$. Extension of haematoma was noted in 3 patients and as associated with a $75 \%$ mortality rate akin to the study done by Fliboutti $\mathrm{JJ}$ et $\mathrm{al}^{20}$.

Infra-tentorial location of the bleed was noted in none of our patient. Though this location of a bleed has been traditionally identified with a worse prognosis. Of the patients with ICH score, score of 0,1 had a good outcome, where as patients with a score of 2 or more had a $90 \%$ chance of a poor outcome. Thus, the ICH score can be used for both risk stratification of patients for better intensive management as well as for prognostication of outcome.

In this study, the ICH-GS scale was found to show a linear highly significant association $(\mathbf{p}<\mathbf{0 . 0 0 1})$ with the grades of outcome as assessed by the modified Ranking Score. Death or severe disability was noted in $10 \%, 15 \%, 55 \%, 90 \%$, almost $100 \%$ of the patients with an ICH-GS score of 5,6,7,8 and more than 9 respectively. At the outset, the ICH-GS might seem more elaborate as compared to the ICH score but since the parameters are the same, and the results better, ICH-GS might be a better scale to adopt in the emergency room.

\section{Conclusion}

The risk factors as identified in the study are increasing age and hypertension.Siriraj score and Allen score were able to identify haemorrhages based on clinical details in $58 \%$ and $50 \%$ of patients, making the scores not reliable enough to replace the CT.Age, systolic blood pressure on admission, GCS, NIHSS on admission, volume of bleed, intra-ventricular extension were all identified as independent predictors of outcome.The (ICH) score and (ICH-GS) are simple tools that could be used for risk stratification as well as prognostication of outcome.

\section{Further research}

Similar studies in larger population to identify epidemiological trends, and consolidate the ICH and ICH-GS Scores as outcome predictors. Application of Essen Intracerebral haemorrhage score49 and FUNC Score50 in predicting the functional outcome after haemorrhage in our population.

\section{Bibliography}

[1]. Sutherland GR, Auer RN. Primary intracerebral haemorrhage. J Clin Neurosci. 2006;13:511-7

[2]. Nilsson OG, Lindgren A, Brandt L, Saveland H. Prediction of death in patients with primary intracerebral hemorrhage: A prospective study of a defined population. J Neurosurg. 2002;97:531-6.[PubMed] 
Associating factors with outcome (Modified Ranking Scale) of spontaneous Intracerebral...

[3]. Hemphill JC, 3rd, Bonovich DC, Besmertis L, Manley GT, Johnston SC. The ICH score: A simple, reliable grading scale for intracerebral hemorrhage. Stroke. 2001;32:891-7. [P

[4]. Medical considerations of Intracerebral Haemorrhage, Stroke chap 58 pg (1359-1388)

[5]. Wade S Smith. Cerebrovascular diseases. Harrison's Principles of Internal Medicine 2513-2525

[6]. Adam's and Victor's principles of Neurology. $8^{\text {th }}$ edition, Cerebrovascular diseases. Chap 34 (718 - 724)

[7]. Epidemiology of stroke. Stroke, Chap 1(3-45); chap 58 (1359-388).

[8]. Arboix et al. Diabetes Mellitus - independent risk factors for mortality of spontaneous intracerbral haemorrhage. Diabetes Care 2000 Oct; 23(10):1527-1532.

[9]. Kocher et al. Poor diagnostic accuracy and applicability of Siriraj score and Allen score. JAPI 2000; 48:584-585.

[10]. Accuracy of 2 scores in the diagnosis of stroke subtypes in multicenter cohort study. Ann Emergency Medicine, 2008.

[11]. Poungavarin N, Vriyavejakul A, Siriraj stroke score and validation study to distinguish between haemorrhage and infarct in stroke. BMJ 1991; 1565-1567.

[12]. Lisck DR, Pasteur W. Early presentation of hemispheric ICH, Predictors of outcome and guidelines of treatment allocation. Neurology 1994; 44; 133-139.

[13]. Fieschi C, Carolei A, Fiorelli M, Argentino C. Changing prognosis of primary intracerebral haemorrhage, results of clinical and CT follow up of 104 pts. Stroke 1988; 19:192-195

[14]. Hemphill JC III, Bonovich DC, Besmertis L, Manley GT, Johnston SC. The ICH Score: a simple, reliable grading scale for intracerebral haemorrhage. Stroke 2001; 32: 891-897

[15]. CJ Weir, J Bradford. Prognostic Value of components of GCS following acute stroke. QJ Med 2003; 96:67-94.

[16]. Prasad K, Menon GR. Comparison of the three strategies of verbal scoring of the Glasgow Coma Scale in patients with stroke. Cerebrovascular Diseases 1998; 8:79-85.

[17]. Broderick JP, Brott TG, Duldner JE. Volume of Intracerebral haemorrhage. Powerful and easy to use predictor of 30 day mortality. Stroke 1993; 24:987-993.

[18]. Tuhrim S, Horowitz DR. volume of ventricular blood is an important determinant of outcome in supratentorial haemorrhage. Critical Care Med 1999; 27,617-621.

[19]. Can we predict outcome at presentation in patients with lobar haemorrhage. Cerebravascular Diseases 2001 11(3):183-189.

[20]. Fliboutti JJ, Hegan N. Hematoma expansion and outcome of ICH. Neurology 2004; 63:1059-1064.

Table No 1: Association of MR score with various variable

\begin{tabular}{|c|c|c|c|c|}
\hline MR Score & Total(N=50) & $\square 4($ good $)(\mathrm{N}=23)$ & $>4(\mathrm{bad})(\mathrm{N}=17)$ & P value \\
\hline Male & $28(56 \%)$ & $15(53.57 \%)$ & $13(46.43 \%)$ & \multirow[t]{2}{*}{$0.226 \mathrm{NS}$} \\
\hline Female & $22(44 \%)$ & $8(36.36 \%)$ & $14(63.64 \%)$ & \\
\hline \multicolumn{5}{|l|}{ Age } \\
\hline$<80 \mathrm{Yr}$ & $39(78 \%)$ & $18(46.15 \%)$ & $21(53.85 \%)$ & \multirow[t]{2}{*}{$0.967 \mathrm{~N}$} \\
\hline$>80 \mathrm{Yr}$ & $11(22 \%)$ & $5(45.45 \%)$ & $6(54.54 \%)$ & \\
\hline HTN & $18(36 \%)$ & $8(44.44 \%)$ & $10(55.55 \%)$ & $0.869 \mathrm{NS}$ \\
\hline Smoking & $13(26 \%)$ & $6(46.15 \%)$ & $7(53.84 \%)$ & $0.856 \mathrm{NS}$ \\
\hline Alcohol & $4(8 \%)$ & $3(75 \%)$ & $1(25 \%)$ & $0.322 \mathrm{NS}$ \\
\hline Systolic BP $\leq 160 \mathrm{~mm}$ of $\mathrm{Hg}$ & $6(12 \%)$ & $4(66.67 \%)$ & $2(33.33 \%)$ & $0.409 \mathrm{NS}$ \\
\hline Diastolic BP $\leq 100 \mathrm{~mm}$ of $\mathrm{Hg}$ & $27(54 \%)$ & $14(51.85 \%)$ & $13(48.15 \%)$ & \multirow[t]{2}{*}{$0.368 \mathrm{NS}$} \\
\hline$>100 \mathrm{~mm}$ of $\mathrm{Hg}$ & $23(46 \%)$ & $9(39.13 \%)$ & $14(60.87 \%)$ & \\
\hline Pulse Pressure $\leq 60 \mathrm{~mm}$ of $\mathrm{Hg}$ & $16(32 \%)$ & $8(50 \%)$ & $8(50 \%)$ & \multirow[t]{2}{*}{$0.697 \mathrm{NS}$} \\
\hline$>60 \mathrm{~mm}$ of $\mathrm{Hg}$ & $34(68 \%)$ & $15(44.12 \%)$ & $19(55.88 \%)$ & \\
\hline \multicolumn{5}{|l|}{ Volume $\square$} \\
\hline$<30 \mathrm{ml}$ & $32(64 \%)$ & $22(68.75 \%)$ & $10(31.25 \%)$ & \multirow[t]{2}{*}{$0.001 \mathrm{~S}$} \\
\hline$>30 \mathrm{ml}$ & $18(36 \%)$ & $1(5.56 \%)$ & $17(94.44 \%)$ & \\
\hline
\end{tabular}

Table No :2 Association of the various score with MR score

\begin{tabular}{|c|c|c|c|c|}
\hline \multicolumn{5}{|c|}{ MR Score } \\
\hline GCS & Total & 4 (good) $(\mathrm{N}=23)$ & $>4(\mathrm{bad}) \quad(\mathrm{N}=17)$ & \multirow{4}{*}{$\begin{array}{l}\text { P value LS } \\
0.001 \mathrm{~S}\end{array}$} \\
\hline 3 to 8 & 19 & 0 & $19(100 \%)$ & \\
\hline 8 to 12 & 13 & $5(38.46 \%)$ & $8(61.54 \%)$ & \\
\hline 12 to 15 & 18 & $18(100 \%)$ & 0 & \\
\hline \multicolumn{5}{|c|}{ ICH Score } \\
\hline 0 & 8 & $8(100 \%)$ & 0 & \multirow[t]{2}{*}{$0.001 \mathrm{~S}$} \\
\hline$\geq 1$ & 42 & $15(35.71 \%)$ & $27(64.29 \%)$ & \\
\hline \multicolumn{5}{|c|}{ ICH-GS } \\
\hline$\leq 6$ & 17 & $15(88.2 \%)$ & $2(11.8 \%)$ & \multirow[t]{2}{*}{$<0.001 \mathrm{~S}$} \\
\hline$>6$ & 33 & $6(19.2 \%)$ & $27(80.8 \%)$ & \\
\hline
\end{tabular}


Figure No 1 Scores wise distribution of the cases

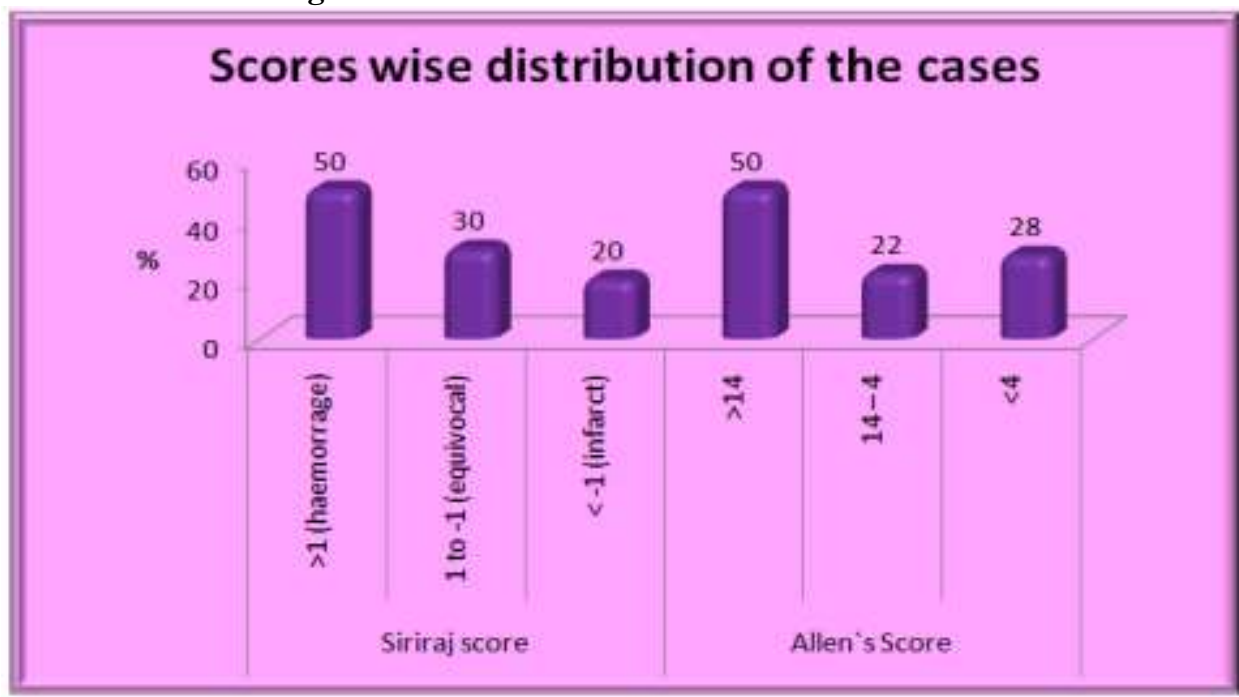

Figure No 2 Complications

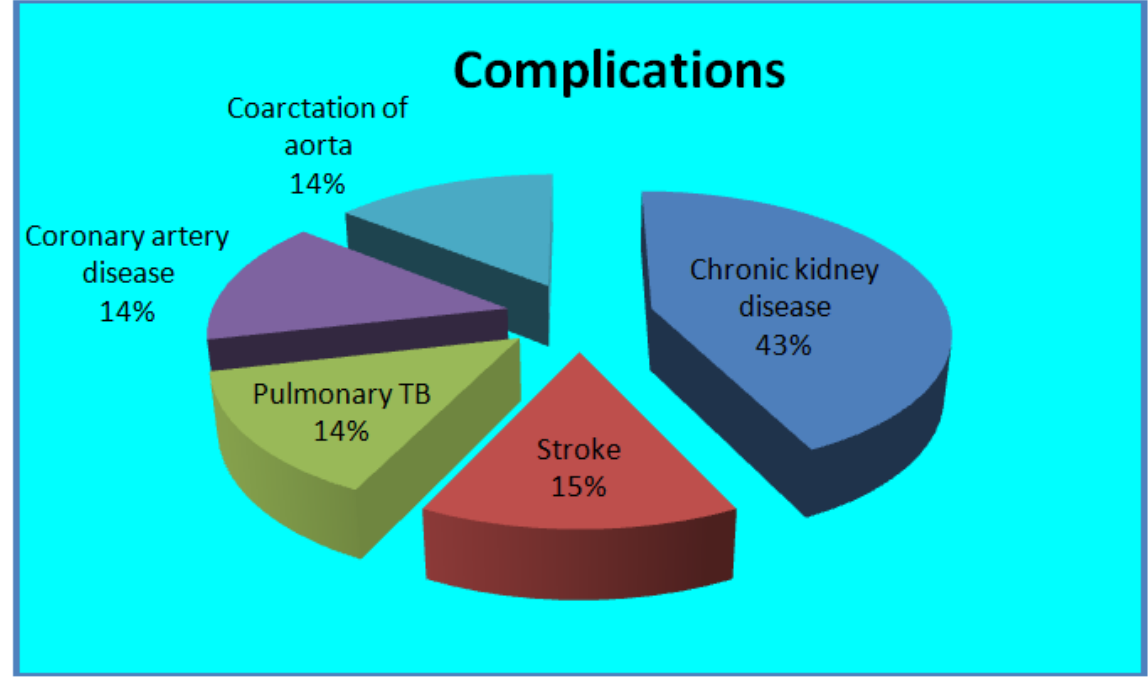

